\title{
Development Trend of Mobile UI Design in Big Data Age
}

\author{
Dong Rui \\ School of Art, Xi'an University, Xi'an, Shanxi, 710065 \\ 397369046@qq.com
}

\begin{abstract}
In contemporary times, mobile Internet technology developed rapidly. Large data information analysis technology has been widely used, and extended to the mobile UI design. User consumption habits, use behavior and other data information has become a guiding factor in mobile UI design, but also an important guarantee to improve the mobile UI interface design value, performance and user experience. Based on the analysis of the new features of user demand in the era of large data, this paper focuses on the innovation of mobile UI design, and then summarizes the future development trend of mobile UI design: pay more attention to user's immersive experience including vision, emotion, custom and dynamic effect.
\end{abstract}

Key words: Large data age; UI interface design; Development trend

\section{Introduction}

In contemporary times, mobile Internet technology developed rapidly. Large data information analysis technology has been widely used, and extended to the mobile UI design. User consumption habits, use behavior and other data information has become a guiding factor in mobile UI design, but also an important guarantee to improve the mobile UI interface design value. The design concept of mobile UI has also changed dramatically, from the user's psychological model as the center into the user experience as the dominant ideology. Product and product interaction, user and product interaction and other interface design immersed experience-based design concept was proposed to promote the changes of mobile UI design methods and design style: simple graphic elements, bright color system, layout hierarchy, Interactive way easy to use and so on.

\section{New Features of User Demand in Big Data Age}

First, the trend of integration is more and more obvious. In today's society, people's living needs are diversified and complicated, which makes the fragmentation feature of mobile terminal users become more and more obvious, including the fragmentation of time, the fragmentation of information and the fragmentation of consumption, which brings some trouble to the users. The Large data information processing technology can effectively deal with fragmented information, including all-round integration of information content, services and use experience. Conduct data analysis and mine user habits and needs, optimize the UI interface design, so that the presentation of information could be more consistent with a user mental model, to improve user's satisfaction with the product ${ }^{[1]}$. Second, the emotional trend is becoming increasingly evident. The society has gradually become the era of emotional consumption, and consumers pay more and more attention to the emotional experience and interpersonal communication when shopping, so the emotional needs 
gradually attracted the attention of businesses. In the design of mobile UI, the designer also needs the analysis of factors including users' physiological and psychological, social and cultural environment, education, and aesthetic preference to make user interface products meet the expected value of the user, produce emotional resonance and interaction between the user and the interface and meet the emotional needs of users. For example, in the interface design of Yixindianzan, when love is magnified, at the same time it will be biased towards the pictures being praised, just like Cupid's sword. This dynamic design makes the interface lively and smart, while easily producing emotional resonance with customers. Third, the trend of personalization and diversification is becoming increasingly prominent. Individuality and diversity have become one of the most important words in modern society. People are paying more and more attention to the pursuit of personalized aesthetics and diversified services. Boot interface of each brand mobile phone reflects the characteristics and cultural connotations of the brand, while each cell phone built-in service software is also more abundant.

\section{Development Innovation of Mobile UI Design in Big Data Age}

\subsection{The minimalism of visual experience}

Minimalism is a major trend in the design of contemporary mobile UI. Vision is the most important way of human perception, more than $80 \%$ of the outside information comes from vision, and the coverage of each mobile APP is very broad, such as Alipay services including online payment, banking, shopping, and information exchange and so on, almost penetrated into every corner of our daily life. Then, in such a complex and diverse interface, allowing users to quickly find the functions they need requires the mobile UI design as concise and intuitive as possible. The details are as follows: the simplification of the interface structure, the simplification of information transmission and the visualization and conciseness of visual presentation. Taking mobile phone Alipay for example, compared to the version 9.2, the show of version 10.0 in the individual function, content and other aspects of the recognition are more direct and concise, and the sweep, pay, transfer, sharing of bicycles in the visual entrance are highlighted. At the same time the icon design is also more concise, abstract and symbolic, such as the icons of the balance of treasure, Amoy ticket are changed into a brief stroke lines from solid, these details change makes Alipay mobile interface more intuitive and simple, user-friendly, and easy to use. Therefore, to obtain simple and effective intelligent operation and reduce user's cognitive load has become an important trend in the development of UI interface design.

\subsection{The freedom and pleasure of emotional aesthetic experience}

Through the ages, artists' artistic creation and aesthetic appreciation can always bring a sense of freedom to those who appreciate it. This sense of freedom is the spiritual and emotional experience that an admirer receives in the face of beauty. When we talk about the "user experience" of UI design, pleasure is the most important keyword, and it is the core of the emotional link between the product and the user. Therefore, in the design of mobile UI, facing the increasingly intense competition of similar products, to get the user's favorite, in addition to improve the function of the product, but also should pay attention to consider the user's psychological expectations and emotion, aesthetic experience. According to the user experience and needs analysis, emotional experience are mainly as follows: the sense of freedom to use, the sense of pleasure to create, visual freshness, the sense of familiarity of operation and so on. At present, mainly through the emotional interaction design to achieve these needs. Emotional interaction is based on meeting the user's emotional needs, 
and uses information as the medium to fuse the emotion between the designer, the product and the user, so that the user can get emotional satisfaction in the process of using[2]. Taking INKS pinball game which won the 2016 Apple Design Award as example, the combination of marbles and painting creation makes the marbles leave a variety of color traces in the movement, when hitting the wall it will "explode" to form a variety of strange but very charming pattern, as if the player are taking the artistic creation. This interface design of game gives the marbles a good artistic and freshness, to bring the user a pleasant gaming experience.

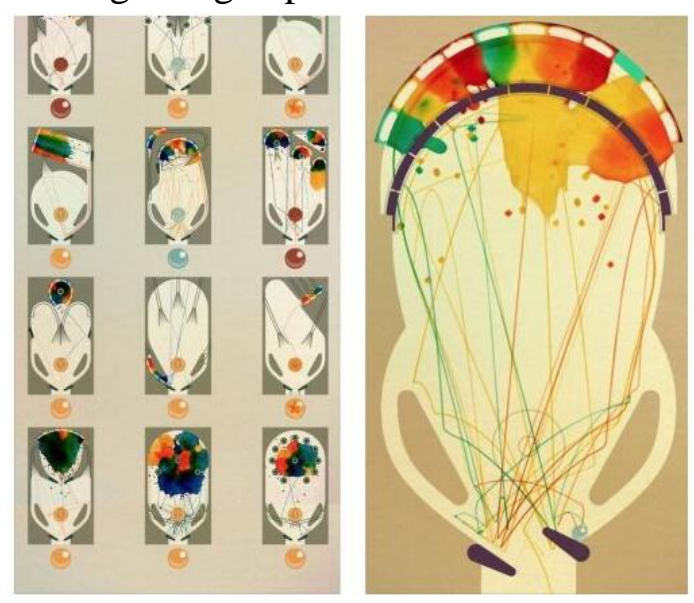

Figure 1 INKS pinball game

\subsection{Personalization and diversification of custom experience}

Customized experience is the most important part of product user experience design, and it is also a major trend in UI design. When using an App or visit a website, users often want to pursuit experience fitting individual needs, but also want to get more diverse information services. As a result, personalization and diversification have become an important requirement for users of large data age. Personalized mobile services provide a wide range of information services based on needs of different users, different needs, and different environments, and change over time and space. WeChat and QQ are two mainstream social APP, and they provide different interface styles according to their customer base. QQ's user base is dominated by young people, mainly for leisure and entertainment. Therefore, it provides personalized customization service specially. The user can choose according to different themes, their preferences, background music player, space, photo albums, fonts, and even when in voice or video chat they could also choose the sound changing effects. In addition, QQ music applications launched a personalized radio station, by analyzing the content of user songs and behavior data, to help users find songs in line with their musical tastes. QQ mobile UI design fits the personality characteristics of teenagers flying themselves and showing their personality. WeChat application software is designed mainly for adults, and the main demand is for work and leisure, so it is very concise in the interface design and function layout, and highlights the core functions. When updating, the maximum it will retain the basic structure and appearance design of product of the last generation, fitting use habit of adults. In addition, the intelligent mobile phone, bracelet, shoes and all kinds of mobile intelligent application software has the function of recording user data, to promote the design of mobile UI visual expression become more personalized, humane and exquisite, to meet user's innovative diverse customized experience. 


\subsection{Mobility integration of mobile UI design}

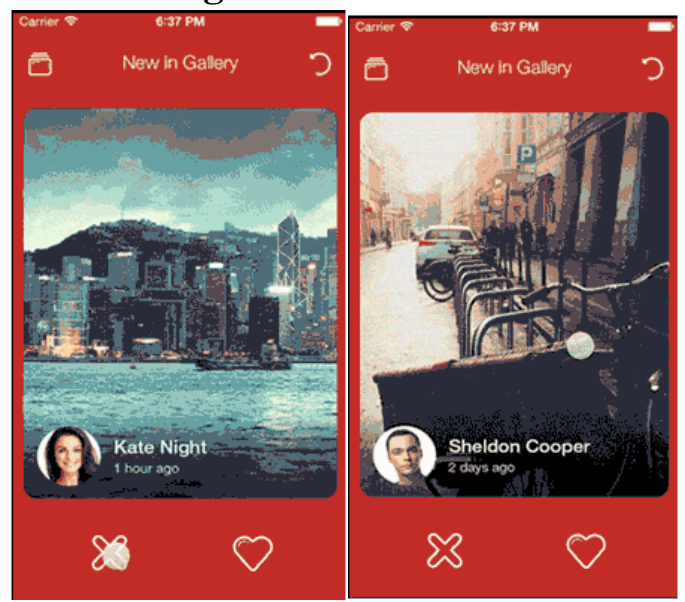

Figure 2 Dynamic effects of Tinder dating apps interface

Originally designed for motion picture and animation, dynamic integration has become an important way to create an excellent user experience in mobile UI design. This is mainly due to the important role of product interaction, including the dynamic feedback effect of interactive gestures such as user's clicks, slides, touches and pull-down, and the introduction and application of animation effects [3]. Dynamic integration can make the interface design more flexible, easier to attract the attention of users, more humane, interesting, and expressive and so on. Taking Tinder dating application software as example, a small white circle slides in the interface up and down, left and right to open different interfaces, in addition to the different logo from top to bottom in the screen, moving right represents pass and moving left represents love.

\section{Conclusion}

The user experience has become the dominant idea of mobile UI interface design. Large data as a new resource, has become a source of new knowledge and create new value, and the continuous innovation and development of the technology can conduct a comprehensive perception, collection, analysis and sharing of data, to provide new way for people to understand and analyze the world, Which provides many possibilities for the expression of innovative function of mobile UI design. It also realizes the interaction between objects and objects and the interaction between people and objects, and becomes an important way to enhance the user experience.

The performance and value of product is the core content of mobile UI interface design at present and even in the future. In order for a mobile application to consistently gain trust from its users, the designer must ensure that every detail of the UI interface design meets user requirements, especially performance and experience.

\section{References}

[1] Lin J Y. Innovation Trend of Mobile Terminal Interface Design in the Big Data Era[J]. Packaging Engineering, 2017.

[2] Lin J Y. Innovation Trend of Mobile Terminal Interface Design in the Big Data Era[J]. Packaging Engineering, 2017.

[3] Kim S. A Study on the UX/UI of Smart Mobile Remote Control App in the Development Trend 
of NUI[J]. Journal of Korea Design Knowledge [디자인지식저널] Vol.29, 2014, null(29):83-92. 